\title{
Cellular level characterization of capillary regression in inflammatory angiogenesis using an in vivo corneal model
}

Beatrice Bourghardt Peebo, Per Fagerholm, Catharina Traneus-Rockert and Neil Lagali

\section{Linköping University Post Print}

N.B.: When citing this work, cite the original article.

The original publication is available at www.springerlink.com:

Beatrice Bourghardt Peebo, Per Fagerholm, Catharina Traneus-Rockert and Neil Lagali, Cellular level characterization of capillary regression in inflammatory angiogenesis using an in vivo corneal model, 2011, Angiogenesis, (14), 3, 393-405.

http://dx.doi.org/10.1007/s10456-011-9223-3

Copyright: Springer Verlag (Germany) http://www.springerlink.com/

Postprint available at: Linköping University Electronic Press http://urn.kb.se/resolve?urn=urn:nbn:se:liu:diva-70325 
Cellular level characterization of capillary regression in inflammatory angiogenesis using an in vivo corneal model.

Beatrice Bourghardt Peebo ${ }^{1,2}$, Per Fagerholm $^{1}$, Catharina Traneus-Röckert ${ }^{3}$ and Neil Lagali ${ }^{1}$

${ }^{1}$ Department of Clinical and Experimental Medicine, Faculty of Health Sciences, Linköping University, 58183 Linköping, Sweden

${ }^{2}$ Department of Ophthalmology/Futurum, County Hospital Ryhov, 55185 Jönköping, Sweden

${ }^{3}$ Department of Pathology, Linköping University Hospital, 58185 Linköping, Sweden

Disclosure: B Bourghardt Peebo, None; P Fagerholm, None; C. Traneus-Röckert, None; N. Lagali, None.

Word count: 4397

Corresponding author and reprint request:

Neil Lagali

Department of Clinical and Experimental Medicine

Faculty of Health Sciences

Linköping University

58183 Linköping

Sweden

Tel: +46 101034658

Fax: +46 101033065

email: neil.lagali@liu.se 


\begin{abstract}
:
In this study, we introduce a technique for repeated, microscopic observation of single regressing capillaries in vivo in inflamed murine corneas. Natural capillary regression was initiated by removal of inflammatory stimulus during an active pro-angiogenic phase, while the additional impact of anti-angiogenic treatment with triamcinolone or bevazicumab was investigated. Capillaries regressed naturally within one week and treatments did not further enhance the natural regression. Morphologically, early-phase regression was characterized by significant lumen narrowing and a significant reduction in CD11b+ myeloid cell infiltration of the extracellular matrix. By one week, vascular remodeling occurred concomitant with $\mathrm{CD} 11 \mathrm{~b}+\mathrm{CD} 68+\mathrm{KiM} 2 \mathrm{R}+$ mature macrophage localization on capillary walls. Empty conduits without blood flow, positive for collagen IV and devoid of vascular endothelium and pericytes, were apparent in vivo and by three weeks were more numerous than perfused capillaries. By three weeks, macrophages aggregated around remaining perfused capillaries and were observed in apposition with degrading capillary segments. Abrupt termination of capillary sprouting in our regression model further revealed vascular endothelial abandonment of sprout tips and perfused capillary loop formation within a single angiogenic sprout, possibly as an intussusceptive response to cessation of the stimulus. Finally, we observed lumen constriction and macrophage localization on capillary walls in vivo in a clinical case of corneal capillary regression that paralleled findings in our murine model.
\end{abstract}




\section{Introduction:}

Capillary regression is a frequent occurrence in a number of tissues and has been studied in several models including development [1, 2], wound healing [3, 4], oxygen induced retinopathy (OIR) [5-7], ovulation [8] and tumors [9]. The cornea, by virtue of its normal avascularity, transparency, and its robust angiogenic response under appropriate conditions, has also been used as a model tissue to study regression [10-13]. Using histology and electron microscopy of corneal tissue sections in tumor and inflammatory angiogenesis models, Ausprunk and co-workers described a sequence of events including degradation of vascular endothelium and removal of vascular debris by macrophages. Macrophages have also been shown to play a key role in other non-inflammatory models of capillary regression [1, 2, 5-7] by initiating degradation of vascular endothelium. Although vascular endothelium is believed to be digested by macrophages, remnants of basement membrane from regressing capillaries have been shown to persist, constituting so-called 'string' or 'ghost' vessels in various tissues $[10,14]$

Capillary regression is also of interest from a clinical perspective. Therapeutically-induced vascular regression has become a common treatment option for pathologic angiogenesis in tumors, eye diseases, and inflammation. Steroids have long been used to promote the regression of undesirable neovessels and are well known for their anti-inflammatory and antiangiogenic effect [15-17]. More recently, bevacizumab, a monoclonal VEGF-A antibody approved for the treatment of metastatic colorectal cancer, has been widely used off-label to successfully induce regression in eye-related angiogenesis in cases of choroidal, retinal and corneal neovascularization in patients [18-24].

In both clinical and experimental studies, corneal capillary regression is commonly evaluated by degree of vascular invasion at the tissue level, using photography of visible blood vessels, 
fluorescein angiography, or by immunostaining of vascular endothelium at fixed time points [17, 21-32]. Little, however, is known about the morphologic characteristics of regressing capillaries at the cellular level following therapy. Furthermore, it has not been possible to follow the same regressing vessels over time with cellular level detail in an in vivo setting.

We have recently developed a model of inflammatory angiogenesis in the rat cornea and a technique whereby single angiogenic vessels are studied in a time lapse manner [33]. The in vivo model preserves the complexity of the physiologic milieu which includes tissue proteins, inflammatory cells, mesenchymal cells, pre-existing vessels and the circulation, all of which may contribute factors regulating the growth and regression of vessels. In this study, we applied our model and technique to characterize the morphology of regression in vivo, in a manner allowing vessels and cell-vessel interactions to be observed over time in the same tissue. Specifically, we sought to i) examine temporal changes in the morphology of vessels in natural and therapeutically-induced regression, ii) track the presence of inflammatory, myeloid-lineage cells [33] during regression, and iii) examine the post-regression microstructure of the affected tissue. In our model, we abruptly terminated angiogenesis in a strongly pro-angiogenic inflammatory environment, relatively early during the course of inflammation. By this approach we sought to investigate how regression occurs in an active sprouting phase, and the potential spatio-temporal role of tissue macrophages in this process. Finally, we investigated whether findings from our in vivo murine model reflected a similar situation of therapeutically-induced capillary regression in human corneal tissue.

\section{Materials \& Methods:}

\section{Animals and induction of inflammatory angiogenesis}

20 twelve- to sixteen-week-old male Wistar rats weighing 200 to 400g (Scanbur AB, Sollentuna, Sweden) were used. All animals were treated following the Association for 
Research in Vision and Ophthalmology (ARVO) guidelines for the Use of Animals in Ophthalmic and Vision Research. With approval from the Linköping regional animal ethics review board, eighteen rats were anesthetized using intraperitoneal injection of dexmeditomedine (Orion Pharma AB, Sollentuna, Sweden) and xylazine (Pfizer AB, Sollentuna, Sweden) and after topical administration of $1 \%$ tetracaine (Chauvin Pharmaceuticals Ltd, London, England) a corneal stromal suture (10-0 nylon) was placed 1.5 $\mathrm{mm}$ from the temporal limbus as previously described [34]. A second stromal suture was placed at the 3 o'clock position, $1.5 \mathrm{~mm}$ from the nasal limbus, to provide additional tissue samples for ex vivo analysis. Left eyes were left untouched and served as negative controls. In a separate experiment, to explore the effect of subconjuntival injection itself, two non-sutured rats were injected with $0.05 \mathrm{ml}$ saline in the right eye under general anesthesia, and the corneas were observed after 24 hours.

\section{Initiation of capillary regression}

Seven days after suture placement, the 18 rats were anesthetized and laser scanning in vivo confocal microscopy (IVCM) of the corneas was performed after topical administration of $1 \%$ tetracaine. The non-invasive IVCM examination lasted for a maximum of 10 minutes, during which time corneas were hydrated with Viscotears (Novartis Healthcare A/S, Copenhagen, Denmark). Immediately after the examination, sutures were removed (day 0) and animals were divided into three groups of six rats each. One group was left untreated to study natural regression (control group). Other animals received a single subconjunctival injection of either bevacizumab $\left(0.05 \mathrm{ml}, 25 \mathrm{mg} / \mathrm{ml}\right.$ Avastin $^{\circledR}$, Roche AB, Stockholm, Sweden - bevacizumab group $)$ or triamcinolone $\left(0.05 \mathrm{ml}, 40 \mathrm{mg} / \mathrm{ml} \mathrm{Kenacort}{ }^{\circledR}\right.$ - T, Bristol - Meyers Squibb AB, Bromma, Sweden - triamcinolone group). Treatment was administered directly after suture removal. Anaesthesia was thereafter immediately reversed by subcutaneous injection of 0.1 $\mathrm{ml}$ atipamezol 5mg/ml (Orion Pharma AB, Sollentuna, Sweden). 


\section{In vivo confocal microscopy}

In this study we used laser scanning in vivo confocal microscopy, an instrument clinically approved for use in imaging the eye. This microscope, (Heidelberg Retinal Tomograph 3 with Rostock Corneal Module, Heidelberg Engineering) was equipped with a 63x/0.95 NA waterimmersion objective (Zeiss, Oberkochen, Germany), that provides an en face view of a 400 $\mu \mathrm{m}$ x $400 \mu \mathrm{m}$ corneal area at a selectable corneal depth. Images are formed by endogenous light scatter from tissue structures after excitation by a $670 \mathrm{~nm}$ red laser. The instrument was prepared as for a routine clinical corneal examination according to the manufacturer's instructions. Briefly, a large drop of transparent tear gel (Bausch \& Lomb, Rochester, NY) was placed on the microscope objective lens, and a sterile disposable plastic cap (Tomocap; Heidelberg Engineering, Heidelberg, Germany) was affixed over the gel-coated lens. A second drop of tear gel was placed on the outer surface of the cap, and the gel-coated cap was brought into contact with the cornea in the suture region using the HRT3 manual alignment controls. The focus depth of the HRT3 was initially adjusted to image the corneal epithelial surface, and the lateral and transverse microscope alignments were adjusted to locate the suture in the real-time image display window. Once the suture was located, the focal plane was adjusted axially to locate blood vessels. Digital images were recorded at 5 frames/s, and the probed region was adjusted in lateral, transverse, and axial directions during image capture to locate and follow the path of vessels from the suture area to the limbus. IVCM has been shown to be a valuable tool for imaging of various cells in transparent tissue, noninvasively, in a time-lapse manner, without the need for cell labeling[33,34]. With IVCM it is possible to identify and follow the same structures and thereby observe dynamic processes in live tissue. The technique, however, is limited to transparent tissues, and cells cannot be characterized by marker expression in vivo. 
In vivo follow up

At days 7 and 21 after suture removal, animals were anesthetized, eyes were photographed in the operating microscope, and IVCM was performed. Additionally, to follow the same vessels in a shorter time interval, 4 of 6 rats in the bevacizumab group were anesthetized and examined daily by IVCM after suture placement and on day 1, 2, 5, 7, 12 and 21 after suture removal and bevacizumab injection. Once the suture was located, the microscope field of view $(400 \mu \mathrm{m} X 400 \mu \mathrm{m})$ was translated temporally to locate the limbal region. A typical examination consisted of 10 to 40 image sequences with each sequence containing 100 successive digital image frames. Sequences could be analyzed frame-by-frame or in video mode, played at the image acquisition rate. Identification of the same vessels at different times was achieved by comparison of vessel patterns with previously stored images. After examination, on day 7, anaesthesia was immediately reversed in twelve of the animals. Following examination at day 7 and 21, six and twelve animals, respectively, were euthanized by intracardiac injection of $100 \mathrm{mg} / \mathrm{kg}$ pentobarbital sodium (Apoteket $\mathrm{AB}$, Linköping, Sweden) while under deep anaesthesia. The entire cornea with scleral rim was then excised, and nasal and temporal portions were prepared for flat-mounting.

\section{Analysis of in vivo images}

Corneas were examined in the operating microscope at day 0 (day of suture removal) and at days 7 and 21 to assess regression at the tissue level. The degree of visible blood vessels in the cornea was evaluated from photographs using a grading of 0 (no visible blood vessels), 1 (1 - 5 vessels), 2 (5 - 15 vessels), 3 (15 - 25 vessels), or 4 (over 25 vessels). To quantify lumen diameter, individual IVCM image frames of blood vessels at each time point, from each animal and from clinical observations, were analyzed (ImageJ software, developed by Wayne

Rasband, National Institutes of Health, Bethesda, MD; available at 
http://rsb.info.nih.gov/ij/index.html). Images with distinct vessels with blood flow were selected and, where possible, were at the same corneal depth. Blood vessels had a constant diameter and were measured once per vessel or twice in a loop (once in each connecting vessel). 40 to 90 values were measured in each group and at each time point, for statistical analysis.

To quantify inflammatory cell infiltration around blood vessels, individual IVCM frames were chosen and analyzed by two different observers. To capture the total inflammatory area within each cornea, three images (from the limbus, stroma and suture) were used for each time point and all visible inflammatory cells within the frames were counted. Inflammatory cells were characterized as distinct, hyperreflective, rounded or spindle-shaped structures in the stroma, representing early infiltrating cells. Mature macrophages, appearing later in our model [33] were fewer in number and were not quantified. Images were evaluated semiquantitatively at days 0,7 , and 21 , using a grading of 0 (0-10 cells/frame), 1 (10-30 cells/frame), 2 (30-50 cells/frame), or 3 (more than 50 cells/frame). In 138 images, observers differed by 1 scale point in 20 cases. The mean grade from the two observers was used in the analysis. Within each treatment group and at each time point, the three mean grade values (from limbus, stroma and suture) for all corneas were grouped for statistical analysis.

\section{Immunostaining in flat mounted corneas}

Frozen corneas were thawed and rinsed in PBS; fixed in acetone; rinsed in PBS 3 times; blocked in $10 \%$ normal donkey serum (Jackson ImmunoResearch Europe, Newmarket, UK) and incubated with primary antibodies overnight. The next day, samples were rinsed, blocked, and incubated with secondary antibodies overnight. For double immunostaining, this procedure was repeated. The primary antibodies used were: pan-endothelial marker CD31/PECAM-1 (Santa Cruz Biotechnology Inc. Santa Cruz, CA, USA), myeloid lineage 
marker CD11b (Santa Cruz), mature pan-macrophage marker for rat tissue KiM2R (Abcam PLC , Cambridge, UK), tissue macrophage marker CD68 (Abcam), mature pericyte marker NG2 (Millipore AB, Solna, Sweden) and marker for basement membrane, Collagen IV (Abcam). Secondary antibodies (Jackson) included Dylight 549, Dylight 649 and Dylight 488. All imaging was performed using a laser-scanning confocal fluorescence microscope (Nikon Eclipse E600 or Zeiss LSM 700) equipped with 40x/1.30 NA oil-immersion or 20x/0.75 NA or 10x/0.75 NA objective lenses. Samples were scanned under single or dual laser excitation and a digital camera was used to record images. In all cases, control samples were used and omission of the primary antibody eliminated cell-specific staining.

\section{Statistical analysis}

Statistical analysis of blood vessel lumen diameter and inflammatory cell presence across groups was performed using Student T-test and one-way ANOVA where data was normally distributed and the non-parametric Mann-Whitney rank sum test otherwise. Pairwise multiple comparisons were performed using Dunn's Method. Normality was determined by the Kolmogorov-Smirnov test. All comparisons were performed using statistical software (SigmaStat, Syastat Software Inc. Chicago, IL, USA) and a two-tailed value of $p<0.05$ was considered statistically significant.

\section{Results:}

Rapid natural regression following loss of inflammatory stimulus is not enhanced by antiangiogenic treatment.

Using the standard clinical method of vessel assessment, rat corneas were imaged under the operating microscope at days 0,7 and 21 in control and treated groups. At day 0 all corneas were fully vascularized to the suture (grade 4). Following suture removal and anti-angiogenic 
treatment, by day 7 all corneas were grade 1 or 2 in control and triamcinolone groups, while in the bevacizumab group all corneas were grade 3 . By day 21 , the majority of corneas were grade 0 or 1 in all groups (Fig 1, Table 1). Staining with the pericyte marker NG2 at day 21 revealed isolated, pericyte-covered CD31+ vessels (Fig 2) that correlated with the few remaining perfused vessels seen in the operating microscope (Fig 1).

Table 1. Time course of the regression of perfused blood vessels from photographs. The values indicate the proportion of samples with each vessel grade (0-4) from each group. Numbers in parentheses indicate number of animals.

\begin{tabular}{|c|c|c|c|c|c|}
\hline Grade & 4 & 3 & 2 & 1 & 0 \\
\hline control do (6) & $100 \%$ & & & & \\
\hline control d7 (6) & & & $83 \%$ & $17 \%$ & \\
\hline control d21 (4) & & & & $50 \%$ & $50 \%$ \\
\hline triam cinolone do (6) & $100 \%$ & & & & \\
\hline triam cinolone d7 (6) & & & $50 \%$ & $50 \%$ & \\
\hline triam cinolone d 21 (3) & & & $33 \%$ & & $67 \%$ \\
\hline bevacizumab do (6) & $100 \%$ & & & & \\
\hline bevacizumab d7 (6) & & $100 \%$ & & & \\
\hline bevacizumab d21 (3) & & & & $67 \%$ & $33 \%$ \\
\hline
\end{tabular}

The lumen significantly constricts during the early phase of corneal capillary regression

Measurement of lumen diameter from in vivo corneal images revealed no significant difference in median diameter between groups at day 0 prior to regression. At day 7 , median lumen diameter in all groups was significantly reduced from day 0 ; by $70 \%$ in the control $(\mathrm{p}=$ $<0,001), 64 \%$ in the triamcinolone $(\mathrm{p}=<0,001)$ and $49 \%$ in the bevacizumab group. $(\mathrm{p}=$ 
$<0.001)$ At day 7, median lumen diameter in the bevacizumab group significantly exceeded control and triamcinolone groups $(\mathrm{p}=<0.001$; Table 2). In vivo imaging revealed substantial narrowing of capillary lumens, with thin wire-like strands of blood flow evident by day 21

(Fig. 3). Substantial thickening of capillary walls was also noted in some cases (Fig 3).

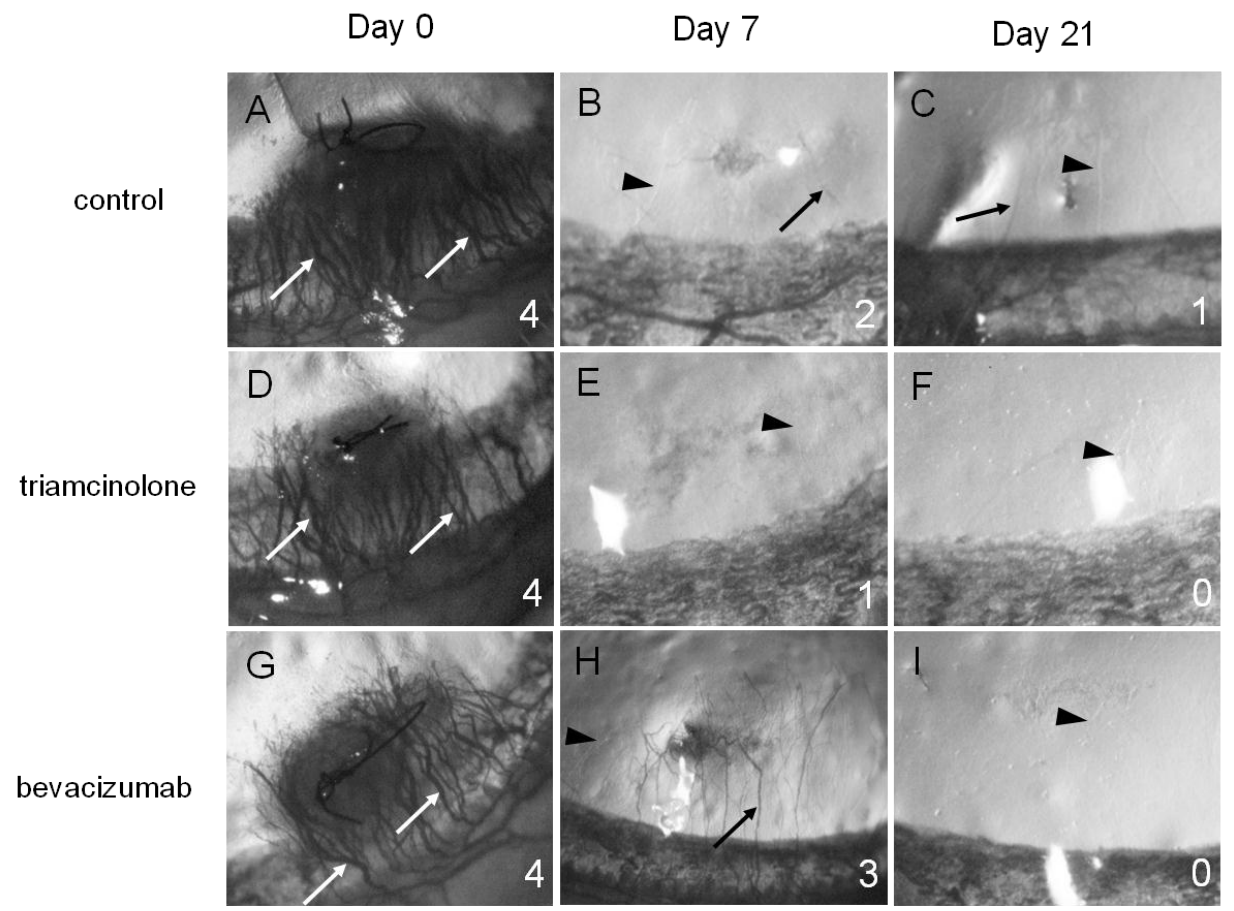

Fig 1. Rapid capillary regression is observed in all groups following removal of inflammatory stimulus from the rat cornea at day 0 . Visible perfused vessels are indicated with white and black arrows. Non-perfused (ghost) vessels (arrowheads) were observed at day 7 and 21 in all groups. The degree of vessel invasion (see methods) is indicated in the lower right hand corner of each image.

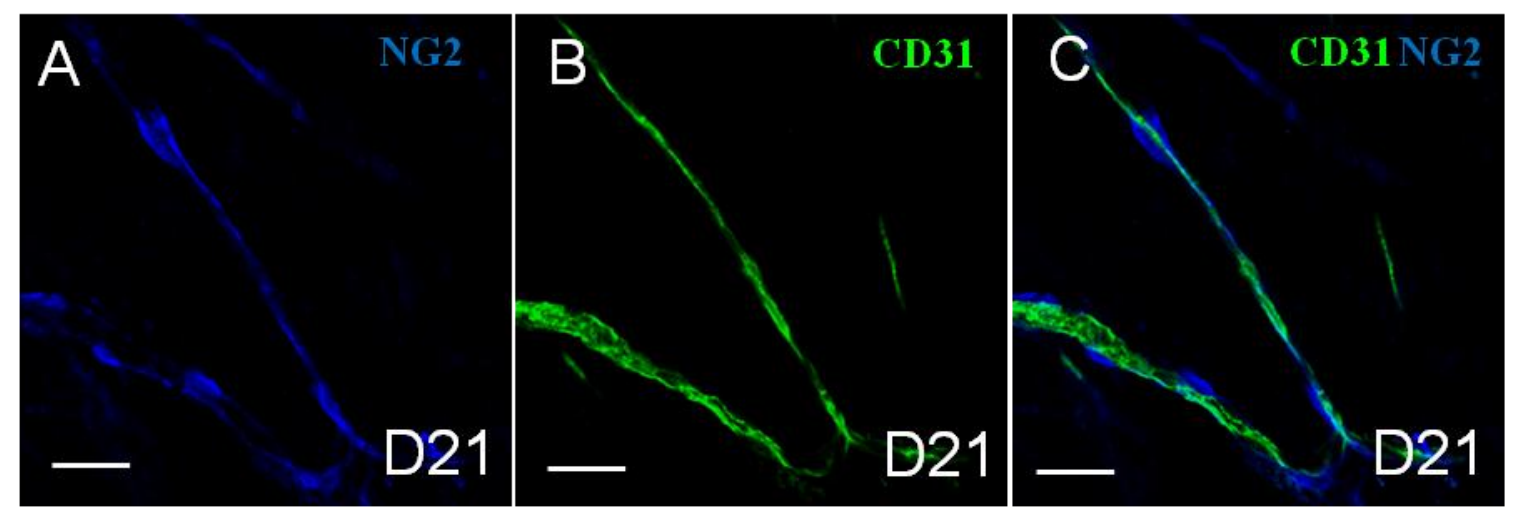

Fig 2. Pericyte-covered vessels 21 days after loss of inflammatory stimulus. A, B and C Expression of CD31 and NG2. (Scale bar $=50 \mu \mathrm{m})$ 
Table 2. Median lumen diameter of perfused blood vessels from IVCM images. Bold values indicate significant inter-group (ANOVA) or within-group (Mann-Whitney) differences. The number of measurements is given in parentheses. ${ }^{a}$ ) median diameter in the bevacizumab group was significantly greater than in control and triamcinolone ${ }^{\mathrm{b}}$ ) median diameter in the bevacizumab group was significantly greater than in control.
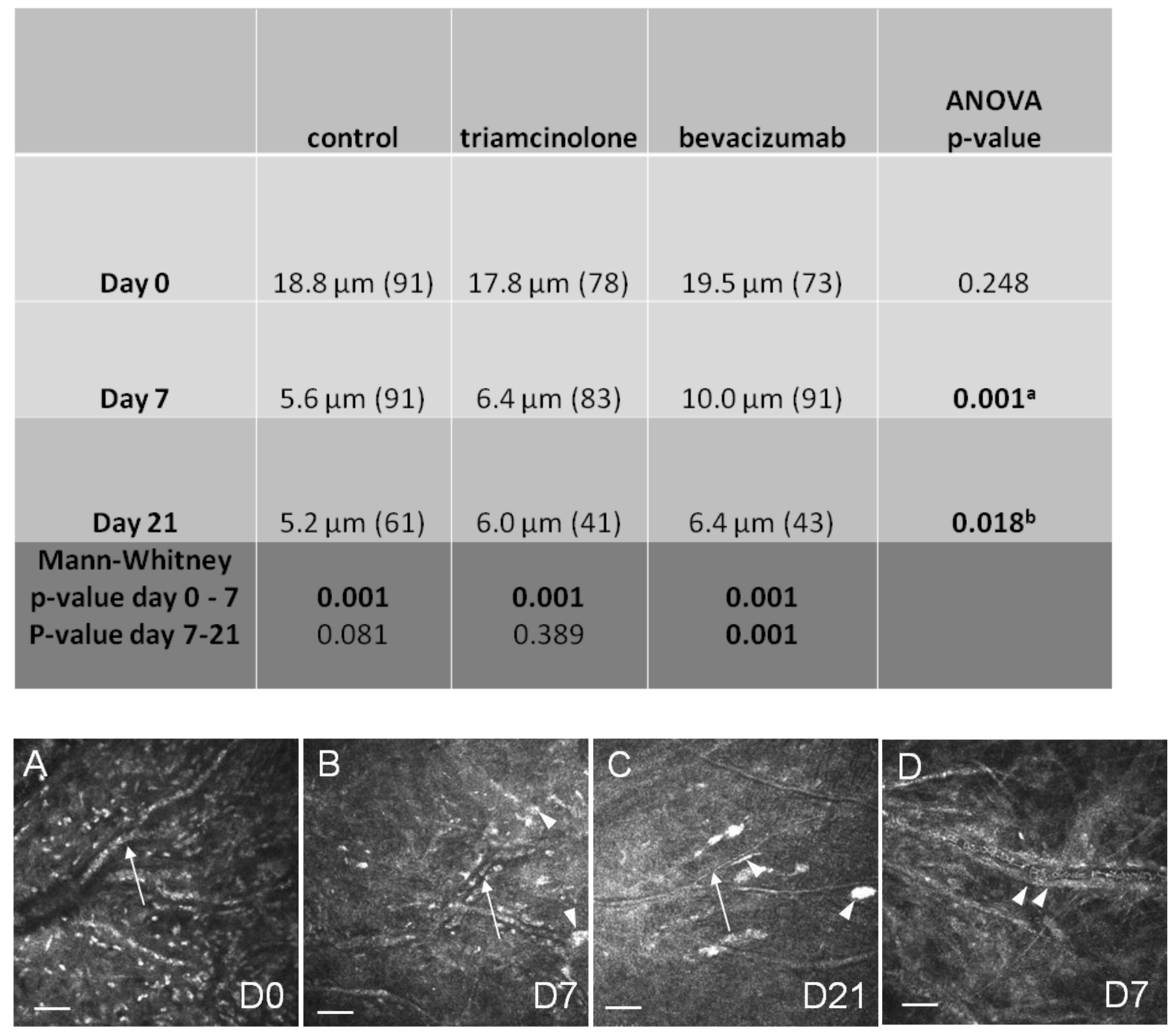

Fig 3. In vivo observations of regressing corneal vessels from day 0 to day 21 . A, B and $\mathbf{C}$ A reduction in vessel lumen diameter (white arrows) was paralleled by a reduction in surrounding infiltrating inflammatory cells. Macrophages (white arrowheads) are observed in close association with the regressing vessel wall from day 7 and onwards. D Substantial thickening of a vessel wall (white arrowheads) at day 7. (Scale bar $=50 \mu \mathrm{m})$

Effectiveness of bevacizumab in a rat corneal inflammation model 
Since all corneas in the bevacizumab group regressed more slowly than in the other two groups, we conducted a pilot experiment to assess the effectiveness of bevacizumab in our model of suture-induced inflammation. Two rats were sutured and treated with one drop of topical bevacizumab $(5 \mathrm{mg} / \mathrm{ml}) 4$ times daily $[22,24,25,29]$ and were followed for 7 days. Two untreated sutured rats served as controls. The treatment had no visible effect on angiogenic sprouting (Fig 4).

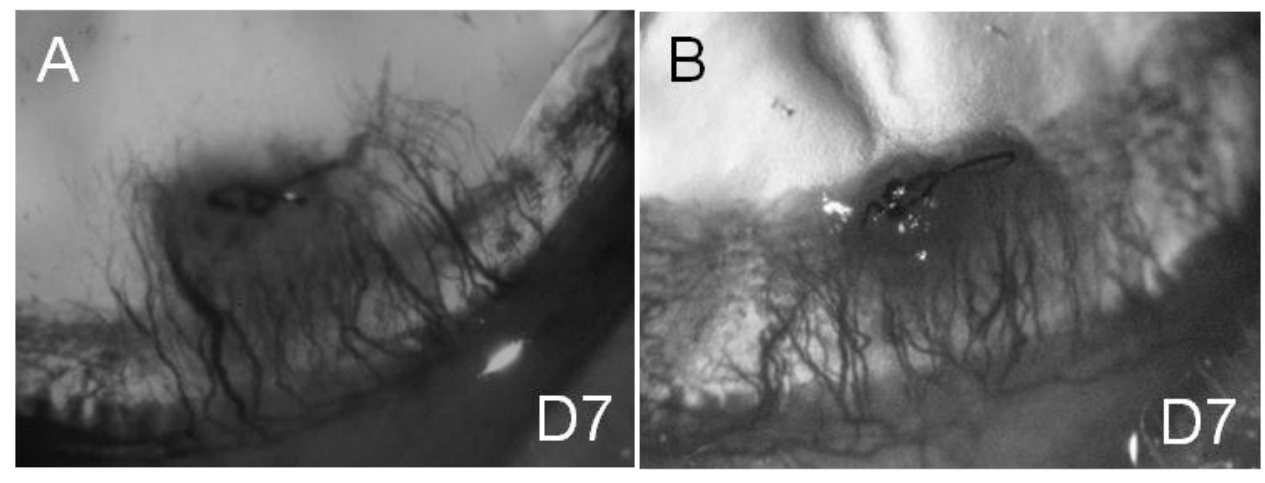

Fig 4. There was no observable effect of topical bevacizumab 4 times daily on inflammatory angiogenesis at day 7. A Control B Bevacizumab

A reduction in infiltrating $C D 11 b+$ myeloid cells parallels the regression of angiogenic vessels

Cells infiltrating the corneal stroma were graded from IVCM image frames taken on days 0,7 and 21 in all corneas (Fig 5 A-C). After semi-quantitative analysis of 138 images, as described in the methods, a significant decrease in inflammatory cell infiltration was found from day 0 to 7 in all groups ( $\mathrm{p}<0,001$ ANOVA), however, no significant change occurred from day 7 to 21 in any group. To determine the presence of macrophages among the inflammatory cells, the myeloid marker CD11b and mature macrophage markers KiM2R and CD68 were used. At day 0, the majority of infiltrating cells were CD11b+, however, a substantial number of CD11b+KiM2R+macrophages were also observed in the stroma (Fig 5 
D). Seven days after loss of inflammatory stimulus, CD11b+KiM2R- and CD11b+KiM2R+ cells both reduced in number (Fig 5E). At day 21, a remaining population of mature CD68+ macrophages was found in close contact with CD31+ vascular endothelium (Fig 5F).

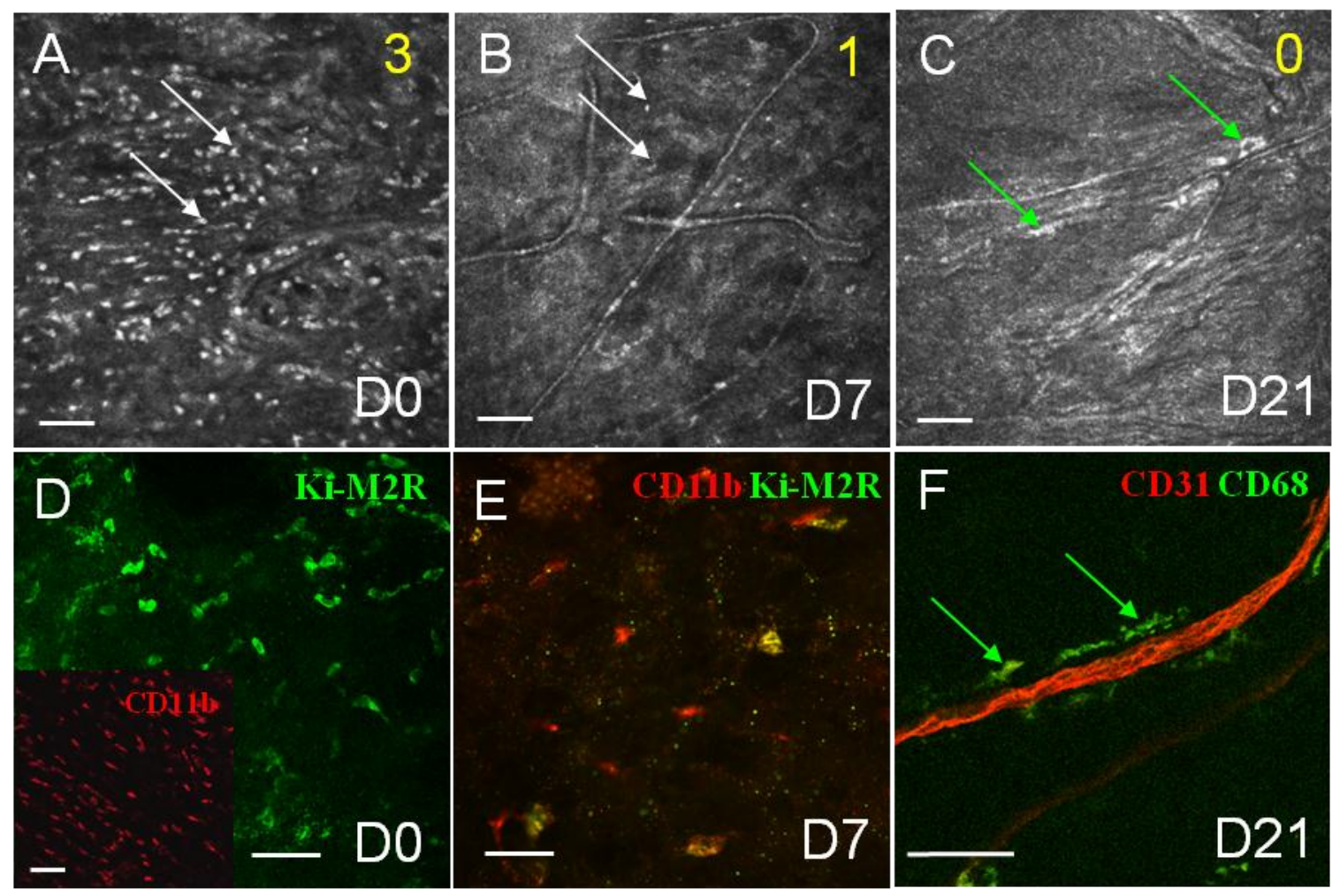

Fig 5. Inflammatory cell infiltration in capillary regression in vivo and ex vivo. A, B and $\mathbf{C}$ A marked reduction of small, rounded hyperreflective inflammatory cells (white arrows) was observed at day 7, while at day 21 remaining cells were macrophages in close association with vessel walls (green arrows). Grade of cell infiltration is indicated by yellow numbers. D At day 0 there was an intense infiltration of CD11b+ (insert) and Ki-M2R+ cells. E At day 7 , the number of myeloid cells and macrophages was reduced. F At day 21, CD68+ macrophages (green arrows) were closely associated with CD31+ vascular endothelium. $($ Scale bar $=50 \mu \mathrm{m})$

Macrophages aggregate at regressing capillary sprout tips and differ in in vivo morphology over time

Prior to initiation of regression at day 0 , inflammatory cells were occasionally observed at capillary walls or sprout tips but did not appear to aggregate. From day 2 onward, however, 
cells were seen aggregating at sprout tips (Fig 6). By day 5, the morphology of these cells in vivo changed from round or elongated with hyper-reflective center to larger, oval-shaped, uniformly diffusely reflective cells. Many cells continued to be associated with sprout tips as well as capillary walls at day 7, during which time capillaries narrowed and vascular remodeling was observed (Fig 6). Characterization of these cells at day 7 revealed that they were mature macrophages (Fig 7). Starting at day 7 and continuing to day 21, macrophages were observed in close relation to vessel stalks, and in many cases had changed in vivo appearance, exhibiting a hyper-reflective cell body with hypo-reflective center (Fig 7). Moreover at day 21, large, contiguous groups of macrophages with this morphology were observed around remaining perfused capillaries at their distal end. Immunohistochemical staining confirmed the in vivo observations of macrophage morphology and aggregation on perfused capillary walls (Fig 7). Proximally at day 21, segmented CD31+ structures, likely representing remnants of vascular endothelium, were observed among capillaries emanating from the limbus (Fig 8). In some cases, macrophages were observed in close apposition with these discontinuous segments.
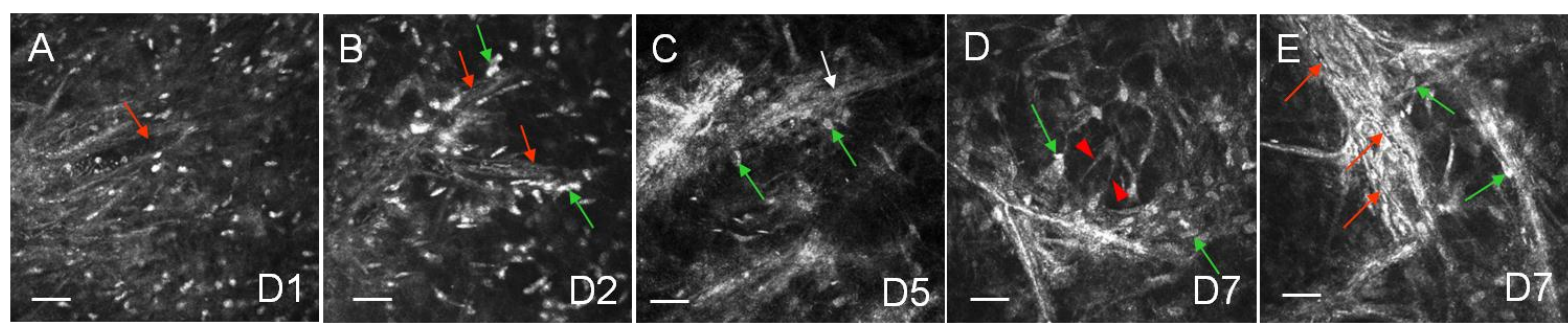

Fig 6. Macrophage contribution to regressing capillary sprouts. A At day 1, perfused sprouts (red arrow) are observed surrounded by round, hyperreflective inflammatory cells. B At day 2, elongated, presumed monocytes or macrophages (green arrows) are observed closely associated with sprout tips (red arrows). $\mathbf{C}$ At day 5, monocytes/macrophages surrounding the sprout tips change morphology to larger, oval-shaped, diffusely reflective cells (green arrows). D Note a macrophages (green arrows) some with pseudopod-like extensions (red arrowheads) extending towards the vessel wall. E Vascular remodeling at day 7. Narrow, perfused capillaries (red arrows) are present within the remodeling sprout, and are surrounded by macrophages (green arrows). (Scale bar $=50 \mu \mathrm{m}$ ) 


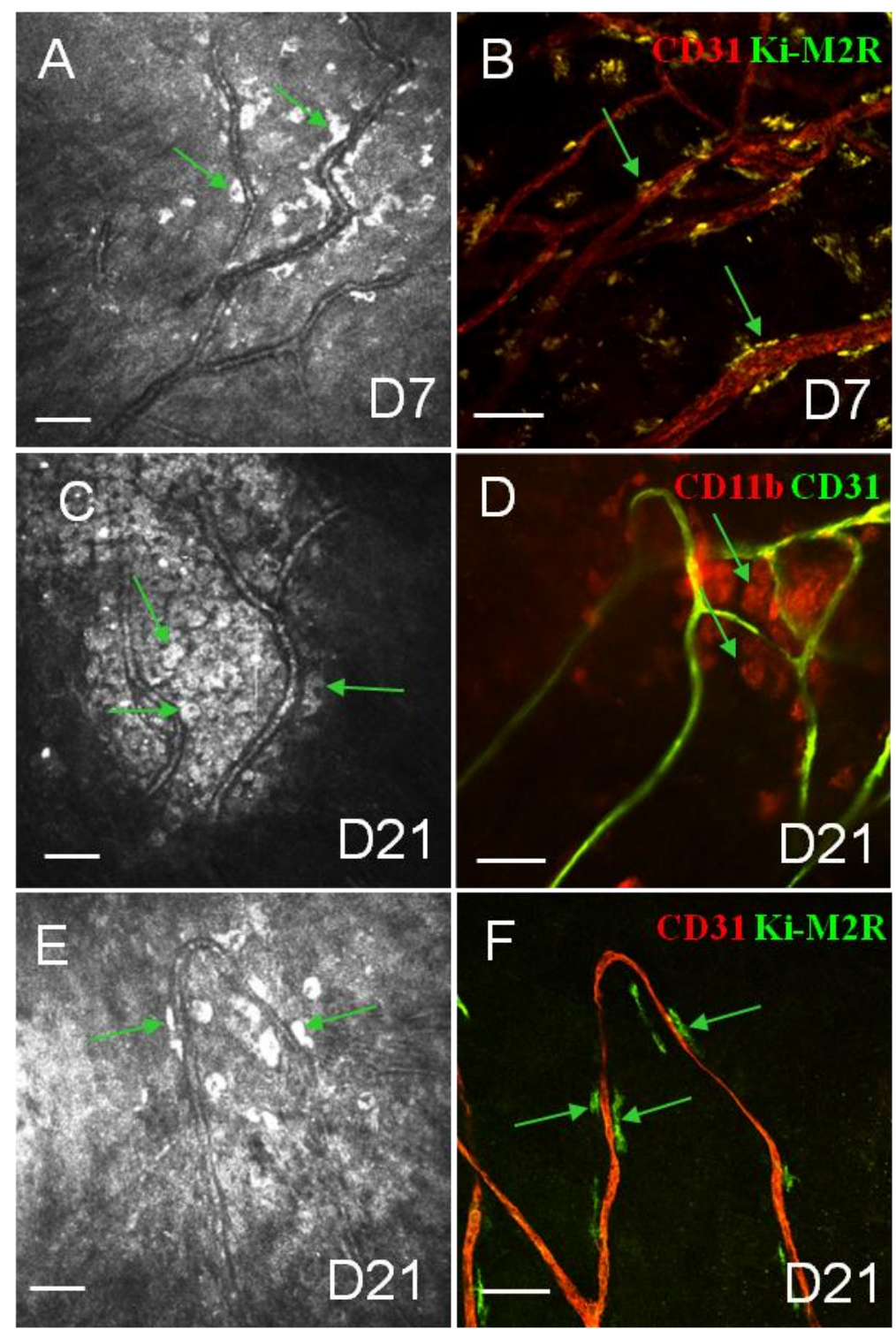

Fig 7. Macrophage contribution to regressing vascular loops and vessel stalks in vivo and ex vivo. A and B At day 7, KiM2R+ macrophages (green arrows) appear attached to CD31+ stromal vessel walls. C and D At day 21, CD11b+KiM2R+ macrophages (green arrows) are observed in clusters and $\mathbf{E}$ and $\mathbf{F}$ individually around perfused CD31+ vessels. (Scale bar = $50 \mu \mathrm{m})$

Angiogenic sprouts remodel into capillary loops during regression

Tracking distal tips of single angiogenic sprouts in vivo on days 5, 7, 12 and 21 revealed the remodeling of a single sprout lumen into a capillary loop, a phenomenon which occurred in all groups (Fig 9). The formation of capillary loops was often seen in combination with vascular pruning. Lumen diameter decreased after loop formation and by day 7 to 12 , sprout tips appeared to be abandoned. The sprout tip remnants were non-perfused in vivo and 


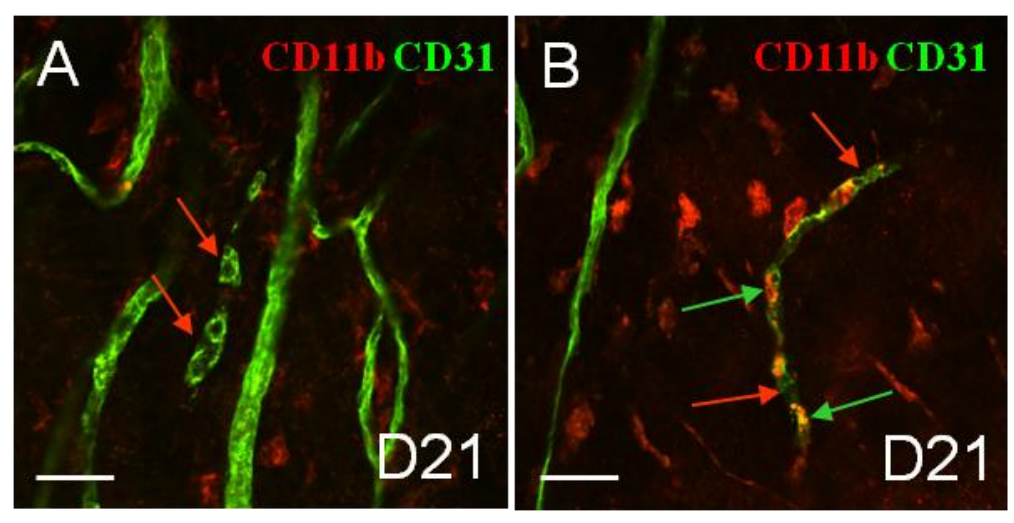

Fig 8. Macrophages contribute to capillary degradation. A Remnants of degrading CD31+ vascular endothelium are indicated by red arrows. B CD11b+ macrophages (green arrows) are seen in close association with a degrading capillary (red arrows). (Scale bar $=50 \mu \mathrm{m}$ )
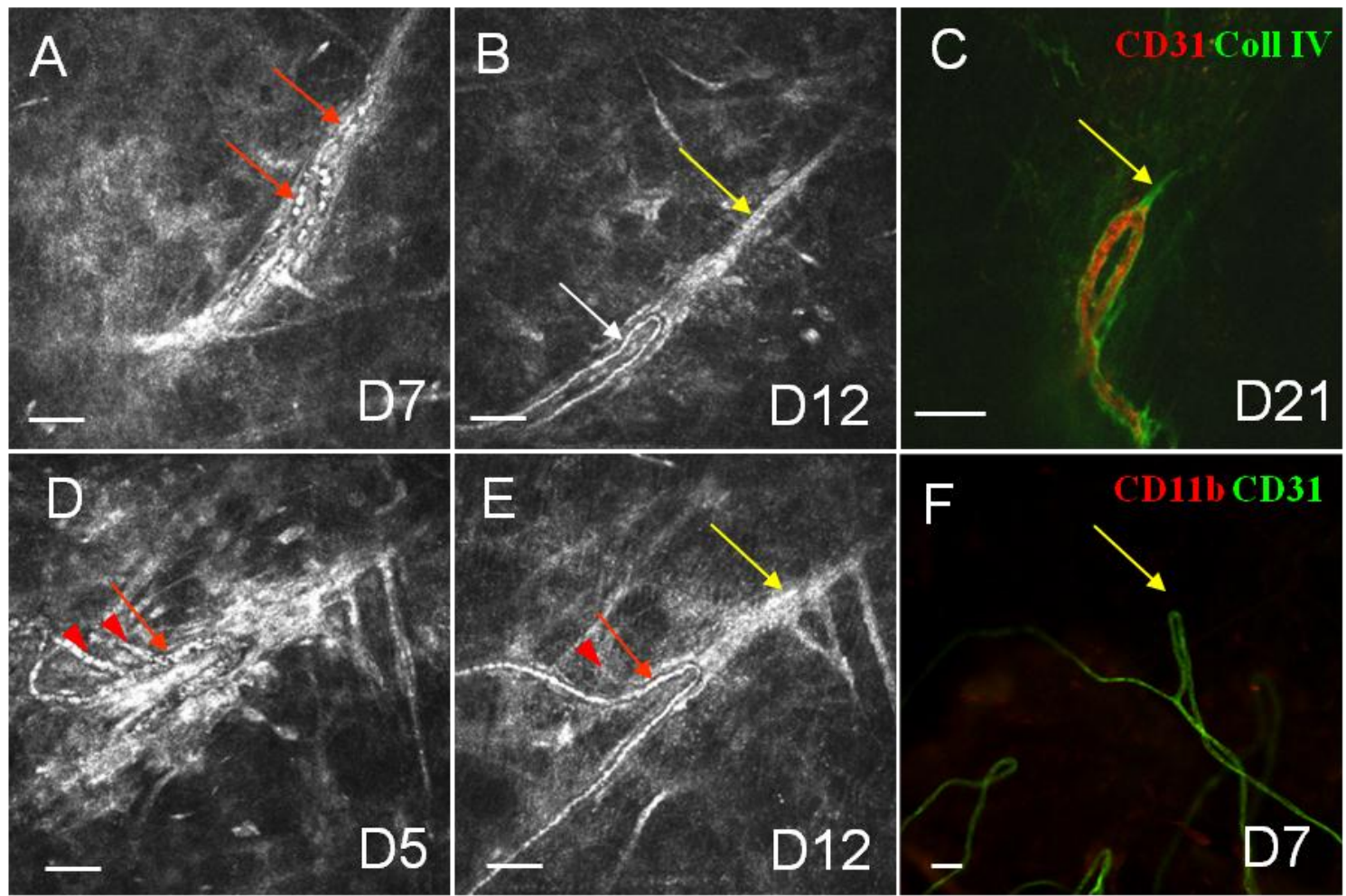

Fig 9. Remodeling of sprout tips in regressing capillaries in vivo and ex vivo. $\mathbf{A}$ and $\mathbf{B}$ Observation of the same sprout at day 7 and 12. A By day 7, the sprout has been remodeled into distinct perfused vessels (red arrows). B At day 12, a vascular loop (white arrow) has formed by pruning of a vessel segment; the sprout tip appears non-perfused (yellow arrow). $\mathbf{C}$ Sprout tips are CD31- and collagen IV+ (yellow arrow) D and E Observation of the same sprout at day 5 and 12. D At day 5, a vascular loop has formed within the sprout (red arrow), and connecting vessels to the loop are observed (red arrowheads). E At day 12, the connecting vessels have been pruned (red arrowhead) and an abandoned sprout tip is observed (yellow arrow) F At day 7, perfused vascular loops at sites of former sprouts are not visible and therefore CD31+ while sprout tips are CD31- (yellow arrow). (Scale bar $=50 \mu \mathrm{m}$ ) 
expressed basement membrane component collagen IV but not vascular endothelial marker CD31 (Fig 9 C,F). Proximally, in vivo observation of the remodeled vasculature revealed perfused capillary loops with a narrow lumen, present within a network of vessel-like structures without visible blood flow (Fig 10A, C). Immunostaining indicated that the nonperfused network was collagen IV+CD31-NG2- while perfused capillary loops were collagen IV+CD31+NG2+ (Fig 10B, D, Fig. 11)
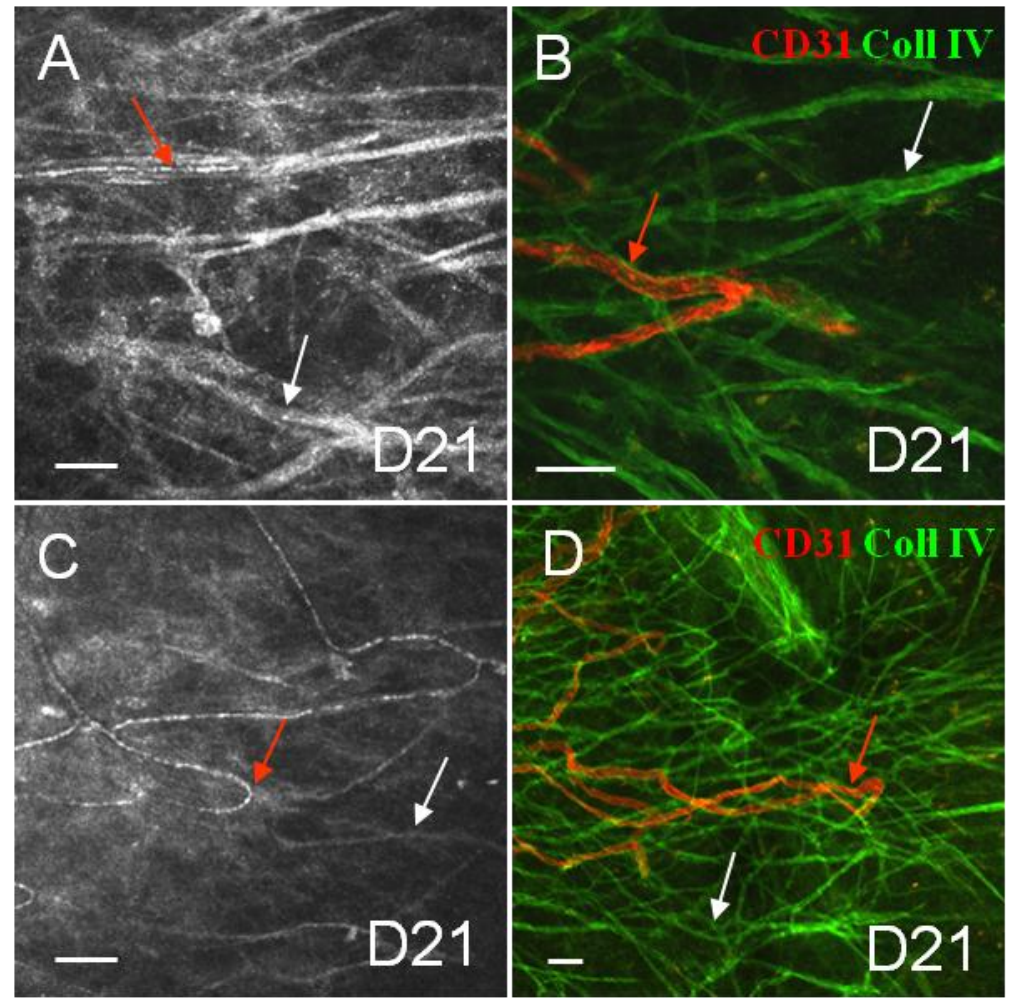

Fig 10. In vivo and ex vivo observation of remodeling of the vascular endothelial network. A and $\mathbf{C}$ At day 21, IVCM revealed a network of non-perfused, diffusely relective vessel-like structures (white arrow), among which some perfused blood vessels were observed (red arrow). B and D The non-perfused network was CD31- and Collagen IV+ (white arrows) whereas perfused vessels were CD31+Collagen IV+ and always terminated in loops. (Scale bar $=50 \mu \mathrm{m})$

Tracking sprouting angiogenesis and regression in a single capillary in vivo

Two days before removal of the inflammatory stimulus, a normally-resident capillary loop was located in a corneal limbal region. The same capillary loop was subsequently tracked by repeated imaging during a two-week period (Fig 12). On subsequent days, lumen expansion 


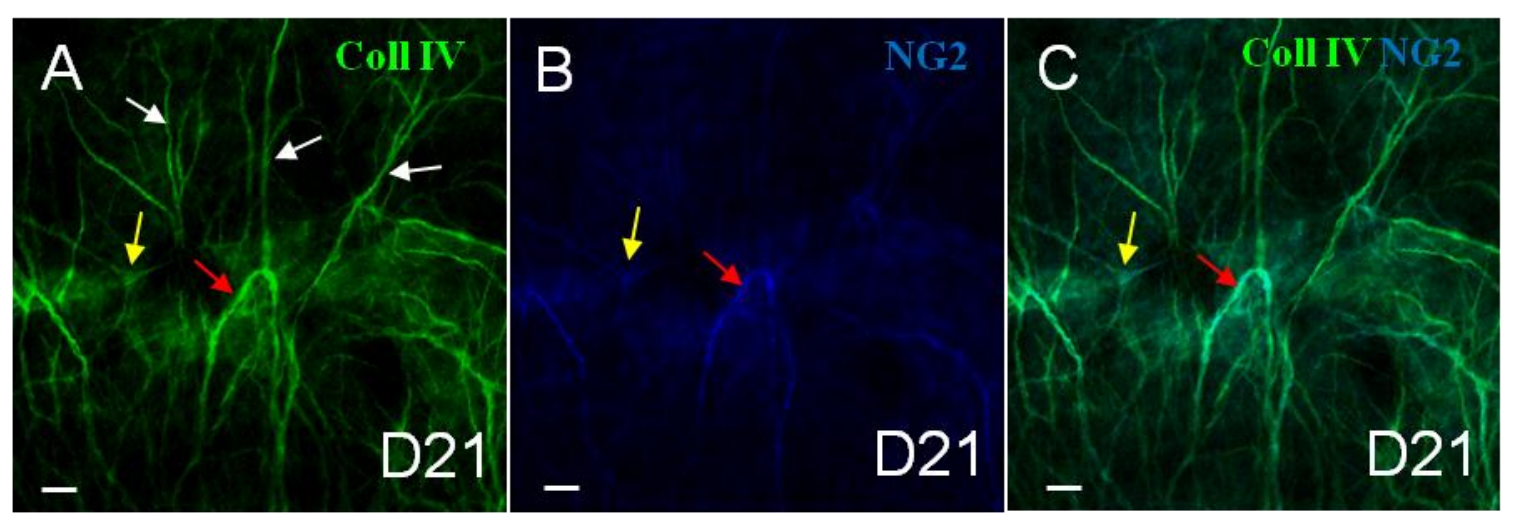

Fig 11. Regression of the vascular network at day 21. A Immature vessels and sprouts (white arrows) and perfused vessels (red arrow) were Collagen IV+. B Perfused vessels (red arrow) were NG2+, while non-perfused structures were NG2-. C Merged image of Collagen IV and NG2. Note that mature, perfused vascular loops (red arrow) expressed basement membrane and pericyte markers. Also note that isolated corneal nerves (yellow arrows), distinguishable by their characteristic branching pattern, also co-expressed collagen IV and NG2.
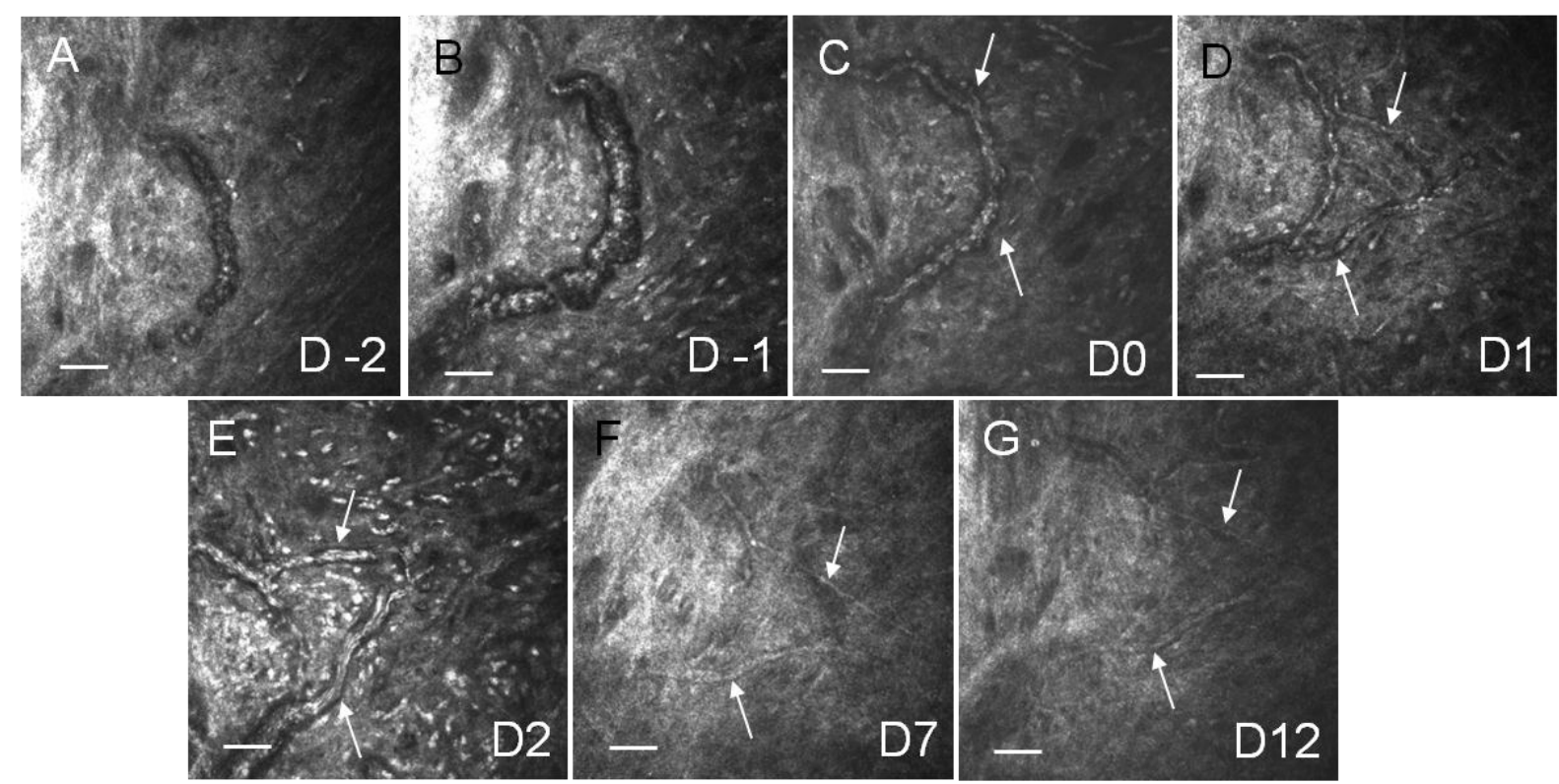

Fig 12. In vivo tracking of sprouting angiogensis and regression in the same capillary. A Two days prior to suture removal, a capillary loop was identified with slight lumen expansion. B One day later, further expansion of lumen diameter was paralleled by vessel wall budding and an increased infiltration of inflammatory cells. C At day 0, new sprouts (arrows) were identified as lumen diameter relaxed. D At day 1, further narrowing of lumen diameter and prominent capillary sprouts were observed (arrows). E At day 2, a new peak of inflammatory cell infiltration was noted, paralleled by expansion of vessel diameter and increased blood flow (arrows). F At day 7, infiltrating inflammatory cells were absent, lumen diameter narrowed (arrows) and flow was profoundly reduced. G. At day 12, a similar appearance as in day 7 was observed. $($ Scale bar $=50 \mu \mathrm{m})$ 
and budding was observed, followed by lumen relaxation and sprouting at budding sites, as previously reported [33]. In the two days following suture removal and bevacizumab treatment, myeloid cells infiltrated the stroma, lumens re-expanded, and blood flow increased. On days 7 and 12, infiltrating inflammatory cells were absent, sprout and feeder lumens were markedly constricted, and flow had diminished considerably (Fig 12).

In vivo imaging of human corneal capillary regression: report of a case

A 55 year old woman with corneal graft failure 30 years after transplantation in the left eye was re-transplanted due to vision-impairing corneal fibrosis secondary to contact lensassociated corneal inflammation. Four months post-operatively, corneal graft rejection occurred and was treated with dexamethasone $0.1 \%$ eye drops (Alcon Sverige AB, Stockholm) hourly, that was slowly tapered. Neovascularization in the graft at the 12 o'clock position at the time of rejection remained after symptoms declined, and was deemed as a risk factor for future rejection. Treatment by a single subconjunctival injection of $0.1 \mathrm{ml}(2.5 \mathrm{mg})$ bevacizumab (Avastin ${ }^{\circledR}$, Roche AB, Stockholm) was given to reduce the neovascularization. On the day of injection (day 0), day 7 and 21, photography and IVCM were performed (Fig 13). By IVCM, median blood vessel lumen diameter was $32 \mu \mathrm{m}(\mathrm{N}=16$ measurements $)$ at day 0 and reduced to $23 \mu \mathrm{m}(\mathrm{N}=14)$ by day 7 ( $28 \%$ reduction, $\mathrm{p}=0.03)$. On day 21 , the median vessel diameter was unchanged from day $7(25 \mu \mathrm{m}(\mathrm{N}=14), \mathrm{p}=0.45)$. In vivo examination of the same vessels confirmed lumen constriction after treatment, while inflammatory cells persisted in the stroma, some of which had a macrophage-like appearance and were located along vessel walls at days 0 and 21 . 

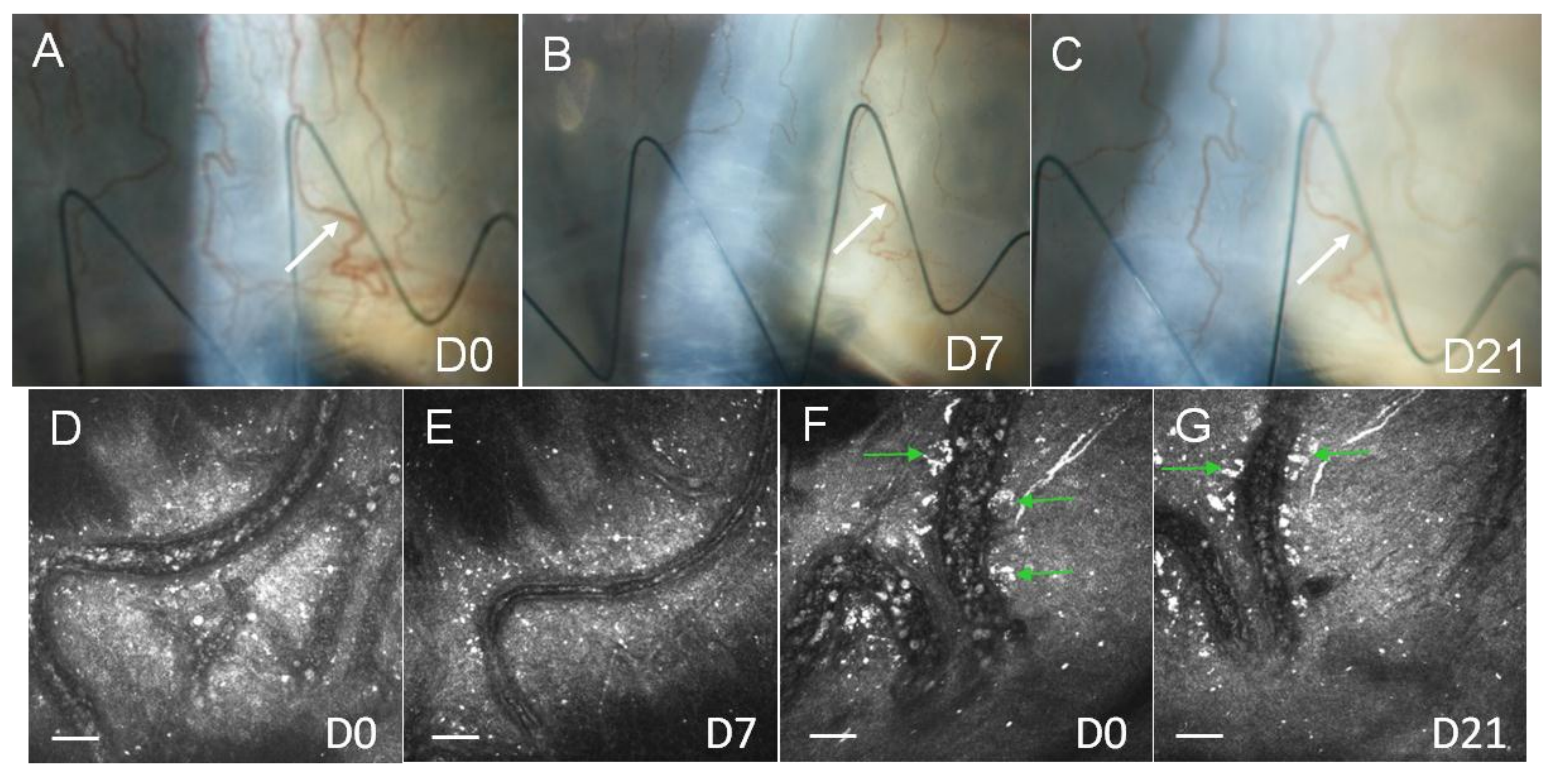

Fig 13. Report of a case with IVCM and photographs. A Appearance of superior corneal graft neovascularization before a single subconjunctival injection of $2.5 \mathrm{mg}$ bevacizumab. B At day 7, blood vessel diameter narrowed. C At day 21 vessel diameter slightly increased. (Arrows in A and C indicate the same blood vessel.) $\mathbf{D}$ and $\mathbf{E}$ Corresponding in vivo images of the same vessel at day 0 and 7. Note a narrowing of the lumen at day 7. F and $\mathbf{G}$ In vivo images of the same vessel at day 0 and 21. At day 21, a narrowing of lumen diameter was observed while macrophages (green arrows) persisted on the vessel wall. (Scale bar $=50 \mu \mathrm{m}$ )

\section{Discussion:}

In this study, several novel features in corneal capillary regression were noted: i) the role of early infiltrating CD11b+ cells in maintaining a pro-angiogenic environment resistive to regression; ii) capillary remodeling by abandonment of sprout tips and capillary loop formation by presumed intussusceptive angiogenesis; and iii) vascular endothelial abandonment of capillaries leaving a network of pericyte free basement membrane tubes. The expected natural regression in an un-stimulated, normally avascular and immune-privileged cornea was confirmed, however, this regression was surprisingly rapid and uninfluenced by additional anti-angiogenic treatment. Delayed regression in the bevacizumab group may have resulted from the local injection itself. In a separate experiment, subjconjunctival injection of 
saline into two healthy rat eyes prompted myeloid cell infiltration into the cornea. The same effect was observed in Fig 12 in the days following injection, and resulted in re-expansion of lumens and stronger blood flow, paralleling the inflammation observed after initial suture placement [33] . Presumably, the broader anti-inflammatory activity of triamcinolone may have aided regression in this group following this second inflammatory insult, compared to a possible lack of VEGFA-specific inhibition by bevacizumab in the other group. We showed, in a separate experiment, that topical bevacizumab had no observable effect on inflammatory sprouting angiogenesis in our model. In the literature there are some controversy concerning the effect of bevacizumab on murine VEGF-A [16, 25, 27, 28, 31, 32, 35]. The effect of bevacizumab on murine VEGF, is possibly route and species dependent $[16,22,25,28,31$, 32].

Correlations we observed between capillary lumen constriction and the absence of early infiltrating myeloid cells (shown in Figs 5, 6, 12) further underscore the importance of these cells in preserving a pro-angiogenic environment. CD11b+ myeloid cells, consisting of populations of neutrophils, monocytes, and macrophages, are known to stimulate and maintain angiogenesis by the release of growth factors [36, 37]. Additional evidence of the role of inflammation in regression was apparent from our case report. Under sustained inflammatory stimulus, inflammatory cells persisted despite bevacizumab treatment, and although treatment induced lumen constriction by day 7 , the constriction was modest and no further constriction (and a possible rebound) was observed on day 21. Taken together, our observations suggest that targeting the early infiltrating CD11b+ myeloid cell population and not only VEGFA may be a more effective strategy [38] in combating pathologic angiogenesis in the eye. 
Within the myeloid cell population, in vivo observation revealed an evolution of some cells into a mature macrophage phenotype that localized near capillary tips and walls, survived the decline of the initial myeloid cell infiltrate, and appeared to take part in vascular remodeling and phagocytosis of vascular endothelial segments. Interestingly, some of these macrophages were observed in vivo with pseudopodia-like extensions connecting to vascular endothelial cells over a distance. These findings are in accordance with earlier reports of macrophages associated with cells that are dying or are destined to be removed from a regressing tissue [1, $2,5,7,10]$. Ausprunk et al. additionally suspected a similar monocyte-to-macrophage transition of cells in close proximity to walls of regressing capillaries [10]. These cells were observed only after regression was initiated and were presumed to originate from the limbal blood vessels. This is in contrast to our earlier findings of CD11b+ myeloid cells extravasating from limbal vessels to infiltrate the corneal stroma shortly after induction of inflammation, with an increasing number of mature macrophages accumulating in the stroma during the pro-angiogenic phase [33]. Our model could be used in the future to investigate the emergence of various macrophage subtypes as the angiogenic balance changes from a pro- to anti-angiogenic environment $[5,7]$.

Significant lumen constriction was noted during the first week of regression in both our rat model and case report, echoing findings in earlier studies [10, 15, 22, 28]. In vivo observation on days 7 and 21, however, revealed more vessels with visible flow than were visible in high magnification photographs. Consequently, in vivo imaging may be a more sensitive method to assess active vessel presence in the cornea after therapy than current methods using photography $[16,17,22,28,30]$ or flourescein angiography $[10,26,29,31]$. At day 21, some persistent vessels were found in the cornea in all groups, and likely reflected their maturity as confirmed by perivascular NG2 staining in this study, and earlier findings of $\alpha \mathrm{SMA}+\mathrm{NG} 2+$ 
cells lining vessels 7 days after stimulus in our model [33]. In another study, Cursiefen et al. noted the persistence of perfused capillaries as long as 8 months after removal of an inflammatory stimulus present for 14 days in the mouse cornea [11]. Compared with the 7day period of inflammation in this study, a longer period can be expected to result in greater pericyte coverage of vessels [13] and their subsequent persistence for an indefinite period.

In this study we followed a sequence of remodeling events that included abandonment of sprout tips, capillary loop formation by presumed intussusceptive angiogenesis, and abandonment of immature capillaries leaving a network of basement membrane tubes, that appeared as 'ghost' vessels under the operating microscope. Capillary loops seemed to have the tendency to resist endothelial cell abandonment, which could be attributed to a preferential stabilization by pericytes or a more robust flow $[2,10]$ compared to distal sprouts, which, although initially perfused, exhibit a more sluggish and intermittent flow, and were not pericyte covered in our model [33]. This result is consistent with an earlier report indicating a stasis of flow preceding platelet sticking and endothelial cell death [10]. Abandoned vessels may provide a scaffold for regenerating capillaries and thereby a route for rapid tissue revascularization. In vivo detection of these remnants in tissue could therefore have a prognostic value.

Our observations of capillary loop formation within a single sprout may indicate a response to the sudden drop in growth factors upon stimulus removal, subsequently promoting vascular endothelial cells to form new vessels without proliferating as an angioadaptive recovery mechanism [39]. Our observations are in accordance with intussusception, where transluminal pillar formation subsequently leads to vessel 'splitting' through vascular endothelial remodeling [40]. Interestingly, we also noted the pruning of vessels in regions of presumed intussusception, an associated sign of intussusceptive angiogenesis [40]. Notably, no evidence 
of intussuception has ever been observed in a pro-angiogenic environment in our model. To our knowledge, this is the first reported evidence of intussusception in inflammatory capillary regression, and the effect may parallel a response seen after tumor anti-angiogenic treatment [41]. As intussusceptive angiogenesis is a short-lived process (occurring over minutes to hours), we did not observe it directly in our model. Future studies with our in vivo time lapse method may reveal more details of this specific event.

In conclusion, we introduce a new in vivo model for characterizing regressing inflammatory capillaries at the cellular level. The method offers the possibility to follow single vessels over time within the complex in vivo environment. Further development of this technique is warranted to investigate in greater detail the local tissue changes accompanying the regression of inflammatory capillaries in a physiologic setting.

\section{References:}

1. Lang RA, Bishop JM (1993) Macrophages are required for cell death and tissue remodeling in the developing mouse eye. Cell 74:453-62

2. Meeson A, Palmer M, Calfon M, Lang R (1996) A relationship between apoptosis and flow during programmed capillary regression is revealed by vital analysis. Development 122:39293938

3. Clark RA (1993) Biology of dermal wound repair. Dermatol Clin 11:647-66

4. Davis GE, Saunders WB (2006) Molecular balance of capillary tube formation versus regression in wound repair:role of matrix metalloproteinases and their inhibitors. J Invest Dermatol 11:44-56. doi:10.1038/sj.jidsymp.5650008

5. Shen J, Xie B, Dong A, Swaim M, Hackett SF, Campochiaro PA (2007) In vivo immunostaining demonstrates macrophages associated with growing and regressing vessels. Invest Ophthalmol Vis Sci 48:4335-4341. doi:10.1167/iovs.07-0113

6. Lobov IB, Sujata R, Carrol TJ et al. (2005) WNT7b mediates macrophage-induced programmed cell death in pattering of the vasculature. Nature 15:417-421. doi:10.1038/nature03928 
7. Davies MH, Stempel AJ, Powers MR (2008) MCP-1deficiency delays regression of pathologic retinal neovascularization in a model of ischemic retinopathy. Invest Ophthalmol Vis Sci 49:4195-4202. doi:10.1167/iovs.07-1491

8. Modlich U, Kaup FJ, Augustin HG (1996) Cyclic angiogenesis and blood vessel regression in the ovary: blood vessel regression during luteolysis involves endothelial cell detachment and vessel occlusion. Lab Invest 74:771-780

9. Hotz B, Buhr HJ, Hotz HG (2008) Intravital microscopic characterization of suramin effects in an orthotopic immunocompetent rat model of pancreatic cancer. J Gastrointest Surg 12:900-906. doi: 10.1007/s11605-008-0507-x

10. Ausprunk DH, Falterman K, Folkman J (1978) The sequence of events in the regression of corneal capillaries. Lab Invest 3: 284-294

11. Cursiefen C, Maruyama K, Jackson DG, Streilen W, Kruse FE (2006) Time course of angiogenesis and lymphangiogenesis after brief corneal inflammation. Cornea 25:443-447. doi: 10.1097/01.ico.0000183485.85636.ff

12. Mwaikambo BR, Sennlaub F, Ong H, Chemtob S, Hardy P (2006) Activation of CD36 inhibits and induces regression of inflammatory corneal neovascularization. Invest Ophthalmol Vis Sci 47:4356-4364. doi:10.1167/iovs.05-1656

13. Jo N, Mailos C, Ju M et al.(2006) Inhibition of platelet-derived growth factor B signaling enhances the efficacy of anti-vascular endothelial growth factor therapy in multiple models of ocular neovascularization. Am J Pathol 168:2036-2053. doi:10.2353/ajpath.2006.050588

14. Brown W (2010) A review of string vessels or collapsed, empty basement membrane tubes. J Alzheimer Dis 21:725-739. doi 10.3233/JAD-2010-100219

15. McKay TI, Gedeon DJ, Vickerman MB, Hylton AG, Ribita D, Olar HH, Kaiser PK, ParsonsWingerter P (2008) Selective inhibition of angiogenesis in small blood vessels and decrease in vessel diameter throughout the vascular tree by triamcinolone acetonide. Invest Ophthalmol Vis sci 49:1184-1190. doi:10.1167/iovs.07-1329

16. Hoffart L, Matonti F, Conrath J, Daniel L, Ridings B, Masson G, Chavane F (2010) Inhibition of corneal neovascularization after alkali burn:comparison of different doses of bevacizumab in monotherapy or associated with dexamethasone. Clin Exp Ophthalmol 38:346-352. doi:10.1111/j.1442-9071.2010.02252.x

17. Kang S, Chung SK (2010) The effect of subconjuntival combined treatment of bevacizumab and triamcinolone acetonide on corneal neovascularization in rabbits. Cornea 29:192-196. doi 10.1097/ICO.0b013e3181b1c82f

18. Subramanian ML, Ness S, Abedi G et al.(2009) Bevacizumab vs ranibizumab for age-related macular degeneration: early results of a prospective double-masked, randomized clinical trial. Am J Ophthalmol 148:875-882. doi:10.1016/j.ajo.2009.07.009

19. Gharbiya M, Giustolisi R, Allievi F, Fantozzi N, Mazzeo L, Scavella V, Balacco Gabrieli C.(2009) Choroidal neovascularization in pathologic myopia: intravitreal ranibizumab versus bevacizumab - a randomized controlled trial. Am J Ophthalmol 149:458-464.

doi:10.1016/j.ajo.2009.10.010 
20. Abdelhakim MASE, Macky TA, Mansour KAG, Mortada HA (2011) Bevacizumab (Avastin) as an adjunct to vitrectomy in the management of severe proliferative diabetic retinopathy: a prospective case series. Ophthalmic Res 45:23-30. doi: 10.1159/000314721

21. Zaki AA, Farid SF (2010) Subconjunctival bevacizumab for corneal neovascularization. Acta Ophthalmol 88:868-871. doi:10.1111/j.1755-3768.2009.0158.x

22. Dastjerdi MH, Al-Arfaj KM, Nallasamy N et al. (2009) Topical bevacizumab in the treatment of corneal neovascularization. Arch Ophthalmol 127:381-389. doi : 0.1001/archophthalmol . 2009.18

23. Bahar I, Kaiserman I, McAllum P, Rootman D, Slomovic A. (2008) Subconjunctival bevacizumab injection for corneal neovascularization. Cornea 27:142-147. doi; 10.1097/ICO.0b013e318159019f

24. Koening Y, Bock F, Horn F, Kruse F, Straub K, Cursiefen C (2009) Short- and long-term safety profile and efficacy of topical bevacizumab (Avastin $\left.{ }^{\circledR}\right)$ eye drops against corneal neovascularization. Graefes Arch Clin Exp Ophthalmol 247:1375-1382. doi 10.1007/s00417-009-1099-1

25. Bock F, Onderka J, Dietrich T et al.(2007) Bevacizumab as a potent inhibitor of inflammatory corneal angiogenesis and lymphangiogenesis. Invest Ophthlamol Vis Sci 48:2545-2552. doi:11.1167/iovs.06-0570

26. Han YS, Lee JE, Jung JW, Lee JS (2009) Inhibitory effects of bevacizumab on angiogenesis and corneal neovascularization. Graefes Arch Clin Exp Ophthalmol 247:541-548. doi:10.1007/s00417-008-0976-3

27. Oh JY, Kim MK, Shin MS, Lee HJ, Lee JH, Wee WR (2009) The anti-inflammatory effect of subconjunctival bevacizumab on chemically burned rat corneas. Current Eye Research 34:8591. doi:10.1080/02713680802607740

28. Dastjerdi M, Saban D, Okanobo A et al.(2010) Effects of topical and subconjunctival bevacizumab in high-risk corneal transplant survival. Invest Ophthalmol Vis Sci 51:24112417. doi:10.1167/iovs.09-3745

29. Pérez-Santonja JJ, Campos-Mollo E, Lledó-Riquelme, Javaloy J, Alió J (2010) Inhibition of corneal neovascularization by topical bevacizumab (anti-VEGF) and sunitinib (ant-VEGF and anti-PDGF) in an animal model. Am J Ophthalmol 150:519-528.

doi:10.1016/j.ajo.2010.04.024

30. Chen WL, Lin CT, Lin NT et al (2009) Subconjunctival injection of bevacizumab (Avastin) on corneal neovascularization in different rabbit models of corneal angiogenesis. Invest Ophthalmol Vis Sci 50:1659-1665. doi:10.1167/iovs.08-1997

31. Drativman-Storobinsky O, Lubin B, Hasanreisoglu M, Goldenberg-Cohen N.(2009) Effect of subconjunctival and intra-ocular bevacizumab injection on angiogenic gene expression levels in a mouse model of corneal neovascularization. Mol Vis 15:2326-2338 
32. Avisar I, Weinberger D, Kremer I. Effect of subconjunctival and intraocular bevacizumab injections on corneal neovascularization in a mouse model.(2010) Curr Eye Res 35:108-115 doi: $10.3109 / 02713680903429007$

33. Bourghardt Peebo B, Fagerholm P, Traneus-Röckert C, Lagali N (2011) Time-lapse in vivo imaging of corneal angiogenesis: the role of inflammatory cells in capillary sprouting. Invest Ophthalmol Vis Sci 52:3060-3068. doi: 10.1167/iovs.10-6101

34. Peebo BB, Fagerholm P, Traneus-Röckert C, Lagali N (2010) Cellular-level characterization of lymph vessels in live, un-labeled corneas by in vivo confocal microscopy. Invest Ophthalmol Vis Sci 51:830-835. doi: 10.1167/iovs.09-4407

35. Yu L, Wu X, Cheng Z et al. (2008) Interaction between bevacizumab and murine VEGF-A: a reassessment. Invets Ophthalmol Vis Sci 49:522-527 doi:10.1167/iovs.07-1175

36. Sunderkötter C, Steinbrink K, Goebeler M, Bhardwaj R, Sorg C (1994) Macrophages and angiogenesis. J Leukocyte Biol. 55:410-422.

37. Capoccia B, Shepherd R M, Link D C (2006) G-CSF and AMD3100 mobilize monocytes into the blood that stimulate angiogenesis in vivo through a paracrine mechanism. Blood 108:2438-2444. doi: 10.1182/blood-2006-04-013755

38. Shojaei F, Wu X, Malik AK et al.(2007) Tumor refractoriness to anti-VEGF treatment is mediated by CD11b+Gr1+ myeloid cells. Nature Biotech 8:911-920. doi:10.1038/nbt1323

39. Hillen F, Griffioen AW (2007) Tumor vascularization: Sprouting angiogenesis and beyond. Cancer Metastais Review 26:489-502. doi: 10.1007/s10555-007-9094-7

40. Makanaya AN, Hlushchuk R, Djonov VG (2008) Intussusceptive angiogenesis and its role in vascular morphogenesis, patterning and remodeling. Angiogenesis 12:113-123. doi:10.1007/s10456-009-9129-5

41. Hlushchuk R, Riesterer O, Baum O, Wood J, Gruber G, Pruschy M, Djonov V (2008) Tumor recovery by angiogenic switch from sprouting to intussusceptive angiogenesis after treatment with PTK787/ZK222584 or ionizing radiation. Am J Path 173:1173-1185.

doi: 10.2353/ajpath.2008.071131 